\title{
ANGLE-CLOSURE GLAUCOMA PRECIPITATED BY THE USE OF PHOSPHOLINE IODIDE FOR ESOTROPIA IN A CHILD* $\dagger$
}

\author{
BY
}

\author{
D. E. P. JONES AND D. M. WATSON \\ St. Thomas's Hospital, London
}

ECOTHIOPATE iodide (phospholine iodide) was introduced in the treatment of glaucoma by Leopold, Gold, and Gold (1957), and has also been used in the treatment of esotropia. Side-effects reported in the use of this miotic for esotropia include iris cysts, blurred vision, and frontal headache (Miller, 1960; Hill and Stromberg, 1962; Abrahamson and Abrahamson, 1964), and diarrhoea and vomiting were reported by Crawford (1964). It has been generally agreed that the local sideeffects occurring when phospholine iodide is used for esotropia are not serious. Iris cysts disappear when the drug is discontinued, while blurred vision and frontal headache are usually mild and transient.

In the following reported case, angle-closure glaucoma in a child was precipitated by the use of phospholine iodide in accommodative esotropia.

\section{Case Report}

A 7-year-old boy originally presented at the age of 3 with an intermittent right convergent squint of the convergence excess type. Atropine refraction showed a total hypermetropia of 4 dioptres. Apart from this and the esotropia, which was only partially accommodative, his ocular condition was stated to be normal in all respects. There was no family history of strabismus or glaucoma. After surgery performed at the age of 4 the esotropia became fully accommodative, but more recently the convergence excess became decompensated, and to help him return to the fullyaccommodative state, he was treated with gutt. phospholine iodide 0.125 per cent. daily to both eyes. This was successful in controlling the esotropia, but during routine examination 7 weeks after starting treatment, it was noticed that both anterior chambers were abnormally shallow.

Examination.-This observation was confirmed by slit-lamp examination, which showed that widespread irido-corneal contact was present in both eyes. The depth of the anterior chamber in the axial region of each eye was estimated as being approximately twice the corneal thickness, that is about $2 \mathrm{~mm}$.

The pupils were not fully miosed and measured 3-4 $\mathrm{mm}$. in diameter in each eye.

The intra-ocular pressures, as measured by applanation tonometry, were $32 \mathrm{~mm}$. $\mathrm{Hg}$ in the right eye and $36 \mathrm{~mm} . \mathrm{Hg}$ in the left.

Both corneae were bright and clear, no epithelial oedema being present. The horizontal corneal diameters were $11.5 \mathrm{~mm}$. in each eye.

No sub-capsular lens opacities or cysts of the iris were present in either eye.

Both optic discs, maculae, and peripheral fundi were normal.

Gonioscopic examination verified the presence of widespread irido-corneal contact in both eyes. In each, however, the neck of the angle sinus was visible below and nasally. In the left eye, none of the trabecular band was visible, whereas in the right eye it was possible to see a small area in the 5 o'clock meridian.

* Received for publication September 26, 1966

$\dagger$ Address for reprints: As above.

59 
Treatment and Clinical Course.-The child was admitted to hospital for rest in bed and atropine ointment 1 per cent. was administered to the left eye only. After 5 hours the pupil in the treated eye had partly dilated and now measured $5 \mathrm{~mm}$. in diameter. In the other eye the pupil had remained at $3 \mathrm{~mm}$. The anterior chamber of the treated eye had deepened appreciably to about twice its previous depth, and no irido-corneal contact was now visible by frontal examination. In the right untreated eye there was little perceptible deepening of the anterior chamber, although the irido-corneal contact was reduced in extent.

In view of the improvement in the treated eye, atropine ointment was then administered to the right eye.

On the next day, nearly 24 hours after the raised tension was discovered, the pupils of both eyes were three-quarters dilated, and the anterior chambers had deepened to about $4 \mathrm{~mm}$. axially. The intra-ocular pressures were $14 \mathrm{~mm} . \mathrm{Hg}$ in the right eye and $17 \mathrm{~mm}$. $\mathrm{Hg}$ in the left, by applanation tonometry.

Follow-up.-After 6 months the intra-ocular pressure remains within normal limits, the latest recording being $12 \mathrm{~mm}$. $\mathrm{Hg}$ in both eyes, by applanation tonometry.

Gonioscopic examination shows that both angles are anatomically medium to wide, and they are both open except where isolated goniosynechiae are present.

\section{Discussion}

The most striking feature when the child was first found to have raised intraocular pressure was the marked decrease in the axial depth of the anterior chamber in each eye. This suggests that the ciliary muscle was in a state of spasm at this time, leading to an increase in the curvature of the lens, and a forward movement of the lens iris diaphragm. It is likely that the ciliary spasm was accompanied by a relative pupillary block and by congestion and swelling of the ciliary body, contributing to the decrease in anterior chamber depth.

Despite the presence of ciliary spasm, the pupils were incompletely miosed. We believe the explanation for this is that adherence of the iris to the posterior corneal surface prevented the production of full miosis.

The rapid return to normal of the depth of the anterior chamber of the left eye after treatment with atropine ointment compared with that of the untreated right eye confirms the essential part played by ciliary spasm in this case.

We have no record of the depth of the anterior chamber or the width of the angles before phospholine iodide was given, but the horizontal corneal diameters are within normal limits (Duke-Elder, 1961), and the intra-ocular pressure has returned to a normal level. Further, in both eyes, there are areas where the angles of the anterior chambers are of normal width and free from goniosynechiae. There is thus nothing to suggest that the eyes were anything but normal for the age of the child. The implication is that those goniosynechiae which were present at the most recent examination, were directly due to the episode of angle closure.

There are no signs of a very prominent "last roll" of the iris, which has been described as an uncommon cause of inverse glaucoma (Higgitt and Smith, 1955), nor is there any evidence of lenticonus.

The severity of the ciliary spasm in this case may be largely due to the relatively high dosage of phospholine iodide, particularly in a patient of this age. Abrahamson and Abrahamson (1964) have recommended that 0.06 per cent. phospholine iodide should be used daily or on alternate days in the treatment of esotropia, stating that at this strength side-effects are negligible. Lloyd (1963) commented that he found 
spasm of accommodation more marked in younger patients on phospholine iodide, and that he preferred to reserve the drug for patients over 55 years of age.

Romano and Jackson (1964) noted that 62.5 per cent. of adult glaucomatous phakic eyes treated with phospholine iodide developed the appearance of iris bombé with an apparent decrease in depth of the anterior chamber. This appearance, which they attributed to ciliary spasm, occurred after variable periods of treatment with phospholine iodide, commonly a few weeks.

We have examined ten further cases of esotropia under treatment with phospholine iodide and have found the intra-ocular tension and the gonioscopic appearance of the angles to be normal in all cases.

\section{Summary}

A case of bilateral angle-closure glaucoma is reported in a child aged 7 years following treatment for accommodative esotropia with phospholine iodide. After treatment with atropine ointment the angles of the anterior chambers re-opened and the intra-ocular pressure returned to normal.

The probable mechanism of this attack is discussed.

We are grateful to Mr. J. Winstanley for permission to publish this case.

\section{REFERENCES}

Abrahamson, I. A., Jr., and Abrahamson, I. A., Sr. (1964). Amer. J. Ophthal., 57, 290. CrAWFORD, R. A. D. (1964). Brit. J. Ophthal., 48, 404.

Duke-Elder, S. (1961). "System of Ophthalmology", vol. 2, p. 93. Kimpton, London. HigGitT, A., and SmITH, R. (1955). Brit. J. Ophthal., 39, 103.

Hill, K., and Stromberg, A. E. (1962). Amer. J. Ophthal., 53, 488.

LeOPOLD, I. H., Gold, P., and Gold, D. (1957). A.M.A. Arch. Ophthal., 58, 363.

LloYd, J. P. F. (1963). Brit. J. Ophthal., 47, 469.

Miller, J. E. (1960). Amer. J. Ophthal., 49, 1350.

Romano, J., and JACKson, H. (1964). Brit. J. Ophthal., 48, 480. 